\title{
Influence on the Accumulation of Anthocyanins by Exogenous ABA and NAA in Cabernet Sauvignon (Vitis Vinifera L.) Grapes and Wines
}

\author{
Jingjing Liu ${ }^{1, ~ a, ~ X i n j i e ~ Z h a o, ~ b, ~ X u e q i a n g ~ G u a n ~}{ }^{2, ~ c}$, Mingyue $\mathrm{Li}^{1, \mathrm{~d}}$ and Xue Yang ${ }^{1, *}$ \\ ${ }^{1}$ Shandong Provincial Key Laboratory of Microbial Engineering, School of Biologic Engineering, Qilu \\ University of Technology (Shandong Academy of Sciences), Jinan 250300 Shandong, China \\ ${ }^{2}$ Institute of Agricultural Products, Shandong Academy of Agricultural Sciences, Jinan 250300 Shandong, \\ China \\ a 1911762066@qq.com, b zhaoxinjie1177@163.com, c guanxq90@126.com, ${ }^{\mathrm{d}}$ 992952881@qq.com \\ * Corresponding authoremail: windy6776@163.com
}

Keywords: Grape; ABA; NAA; Anthocyanins; Wine.

\begin{abstract}
The effects of exogenous abscisic acid (ABA) and naphthylacetic acid (NAA) on phenolic characteristics of grapes and wines were investigated in Cabernet Sauvignon (Vitis Vinifera L.). Exogenous ABA treatment before veraison significantly improved berry and wine attributes. ABA accelerated the accumulation of anthocyanins in grape skins but cannot increase the total anthocyanin content. And NAA significantly inhibited the accumulation of anthocyanins. The wines made from ABA and NAA treated grapes were detected 19 individual anthocyanins. The proportions of acylated anthocyanins and derived pigments in the wines were decreased to some degree by exogenous ABA treatment. And NAA had the opposite effect. Wines with high acylated anthocyanins were more stable in color. These findings provide insight into the effect of ABA and NAA spray on grape and wine anthocyanins, and the stable color of wine.
\end{abstract}

\section{Introduction}

Phenolic compounds have important effect on grape and wine quality due to their key roles in wine color and mouth feel properties, as well as its aging potential and stability. The main classes of phenolic compounds in grape and wines include phenolic acids, stilbenes, and flavonoids (i.e. anthocyanins, flavonols, flavan-3-ol monomers, and proanthocyanidins). These compounds can be extracted into wines during maceration and fermentation.

Anthocyanins are an important secondary metabolite and provide color and antioxidant activity to grape berries and wines [1]. The type, content and proportion of anthocyanins determine the color of grape skin and the color characteristics and aging potential of wine [2-5]. Flavonoids and phenolic acids, known as polyphenols, act as co-pigments to stabilize the color [6]. Anthocyanin sugar molecules can acylate organic acid molecular groups, usually at the C6 position of glucose, and a few also acylate at the $\mathrm{C} 2, \mathrm{C} 3$, and C4 hydroxyl positions to form different acylated monomers [7, 8]. The most common in wine grapes are acetylated, coumalylated, and coffee acylated groups [9]. Some studies have shown that acylated anthocyanins have significant advantages in terms of stability and antioxidant function [10, 11].

The accumulation of anthocyanins starts at the time of veraison, which is the onset of berry ripening [12]. Veraison of grapes can be identified from an increase in soluble solids content, berry softening, and a sudden increase in the skin color [13]. The biosynthesis and accumulation of anthocyanins are affected by many factors, such as grape variety, grape ripeness, environmental factors, viticulture practices and vinification processes, which can qualitatively and quantitatively affect the phenolic composition of the grape and wine and, therefore, their nutritional and quality properties [14].

Various physiological changes involved in the ripening of grape berries are regulated by complex signals of plant hormones, including ethylene, abscisic acid (ABA), brassinosteroids, 
brassinosteroids, jasmonates, polyamines, cytokinins, and auxins (IAA, IBA, naphthaleneacetic acid (NAA), etc.) $[15,16]$. Several studies have demonstrated the multiple applications of exogenous abscisic acid can increase phenolic contents, mainly anthocyanins and flavonols, and antioxidantproperties of the grape skins [17-21]. The wines made from ABA-treated grapes were also enhanced in total phenolics, anthocyanins, flavonols and antioxidant activities [21]. However, the application time and concentration of ABA are critically important for the effective improvement of color development in grape skin [22-24]; this may vary depending on the cultivar and area of application.

Merlot berries treated with naphthylacetic acid (NAA) at pre-veraison showed strongly delay in ripening inception, suppressed accumulation of anthocyanins of berries with down-regulation of genes involved in flavonoid biosynthesis and cell expansion [25]. It is reported NAA application affects the natural decline of IAA in advanced berries and delays the ripening, thereby reducing variance between under-ripe and riper berries of the same cluster [26].

There are many studies on the effects of ABA, GA3, IAA on anthocyanins of grape berries. However, the effects of ABA and NAA on the the types of anthocyanins in wine and wine color has not been studied. Therefore, in this experiment, the effects of exogenous plant growth regulators on the total anthocyanins in grapes and the color oxidation of wines were studied by externally applying ABA and NAA.

\section{Materials and methods}

\subsection{Reagents and Standards}

All solvents were of HPLC quality and all chemicals were of analytical grade ( $>99 \%)$. Commercial standards of Delphinidin-3-O-glucoside, Petunidin-3-O-glucoside, Malvidin-3-O-glucoside were purchased from Sigma-Aldrich (Missouri, USA). Sodium hydroxide $(\mathrm{NaOH})$, phenolphthalein, Folin-Ciocalteu, sodium carbonate $\left(\mathrm{Na}_{2} \mathrm{CO}_{3}\right)$, sodium nitrite $\left(\mathrm{NaNO}_{2}\right)$, aluminum nitrate $\left(\mathrm{Al}\left(\mathrm{NO}_{3}\right)_{3}\right)$, acetone, formic acid, and methanol were provided by Comeo (Tianjin, China). Gallic acid and rutin were purchased from Yuanye (Shanghai, China).

\subsection{Field Treatments}

The experiments were carried out in 2018 using 12-year-old, own-rooted Vitis vinifera plants cv Cabernet Sauvignon in a commercial vineyard located in Xinjiang province, China. Vines were arranged in north-south rows with a between-row and within-row spacing of $2.50 \times 1.0 \mathrm{~m}$, respectively. The vineyard was managed according to the standard viticultural practices for the cultivar and region.

The grapevines of the same age, size, and growth conditions were selected and assigned to receive one of the three treatments: control (water only), $1000 \mathrm{mg} / \mathrm{L} \mathrm{ABA}$ and $200 \mathrm{mg} / \mathrm{L} \mathrm{NAA}$. Spray was done directly on the grapes with a hand-held sprayer until run off 7 days before veraison. Grape berries were collected at the E-L stage of 35, 36, 37 and 38 [27]. The skin of the grapes was manually separated from seeds and pulp and grounded into powder under the protection of liquid nitrogen, and stored in a refrigerator at $-40{ }^{\circ} \mathrm{C}$ for later use. The remaining grapes were used for small-scale vinification.

\subsection{Determination of Berry Weight, Total Soluble Solids (TSS), Titratable Acidity (TA) and PH}

The grape berries were weighed after maturation and average berryweight was recorded. The TSS contents were calculated using a digital refractometer (WYT-III, Haochuang, China) by crushing the berries of eachplot, and the results were expressed in ${ }^{\circ}$ Brix. The titratable acidity was determined by a titrimetric method [28]. $5 \mathrm{ml}$ of fruit juice was titrated with $0.1 \mathrm{M} \mathrm{NaOH}$ to an end-point at $\mathrm{pH} 8.3$ using phenolphthalein as an indicator. The $\mathrm{pH}$ was measured using a $\mathrm{pH}$ metre (PB-10, Sartorius, Germany). 


\subsection{Extraction and Determination of Total Phenolics}

To extract total phenolic content, $1 \mathrm{~g}$ of grape skin or seeds powder was added to $10 \mathrm{~mL}$ of extraction solution (acetone: water: formic acid $=80: 19: 1, \mathrm{v} / \mathrm{v} / \mathrm{v}$ ), extracted in an ultrasonic cleaner for 30min, then centrifuged at $4{ }^{\circ} \mathrm{C} 12000 \mathrm{r}$ for $20 \mathrm{~min}$. The supernatant was separated and the resulting pellet was extracted up to three times using the same volume of the solvent mixture $(10 \mathrm{~mL})$ each time. The supernatants were then combined and and stored at $4{ }^{\circ} \mathrm{C}$ until used. Quantification of total phenolic content in samples was performed by the Folin-Ciocalteu's (FC) reagent [29], using gallic acid as standard. The results were expressed as milligrams of gallic acid equivalents per gram of fresh grape skins (mg of GAE/g).

\subsection{Extraction and Detection of Anthocyanins}

The extraction of anthocyanins follows the following method. For each sample, $1 \mathrm{~g}$ of grape skin powder was added to $10 \mathrm{~mL}$ of extraction solution (acetone: water: formic acid =70: 29: 1, v/v/v). The mixture was kept in dark for $30 \mathrm{~min}$ at $35^{\circ} \mathrm{C}$, and then centrifuged at $4{ }^{\circ} \mathrm{C}, 12000 \mathrm{r}$ for $20 \mathrm{~min}$. The supernatant was collected and used for the quantification of total anthocyanins by aspectrophotometer (Unico7200, Unico, United States) at $520 \mathrm{~nm}$. The results were expressed as milligrams of total anthocyaninsas malvidin-3-glucoside per gram of berry skin (mg.g-1).

For analysis of individual anthocyanins as described by Mattivi [30], the supernatant was evaporated under alow-pressure vacuum (Univapo 100 ECH, Uni Equip, Germany) and the residues were dissolved in $10 \mathrm{~mL}$ of methanol.

\subsection{Analysis of Anthocyanins by UPLC-MS/MS}

Individual anthocyanins were detected with a UPLC-MS/MS System (Waters Acquity UPLC, United States; Bruker Q-TOF-MS, Germany). For the analysis of anthocyanins, the sample (grape extract or wine) was filtered through a $0.22 \mu \mathrm{m}$ PTFE filter prior to injection. And $1 \square \mathrm{L}$ of sample was injected. The column used was a reverse phase (RP) Acquity UPLC BEH C18 $(1.7 \mu \mathrm{m}, 2.1 \mathrm{~mm} \times$ $100 \mathrm{~mm}$, Waters), at $45^{\circ} \mathrm{C}$ with a flow rate of $0.4 \mathrm{~mL} / \mathrm{min}$. And $0.2 \%$ formic acid was used as solvent $\mathrm{A}$ and acetonitrile as solvent $\mathrm{B}$. The elution gradient is as follows: $5 \%-45 \% \mathrm{~B}$ from time 0 to $20 \mathrm{~min}$; 45\% -90\% B from 22 to $22 \mathrm{~min}$; $90 \%$ B from 22 to $26 \mathrm{~min}$; $90 \%-5 \%$ B from 26 to $26.1 \mathrm{~min}$; 5\% B from 26.1 to 30min.MS / MS: Electrospray ion source ESI, positive ion mode; capillary voltage $3.5 \mathrm{Kv}$; sprayer $2.0 \mathrm{Bar}$; dry gas $8.0 \mathrm{~L} / \mathrm{min}$; temperature $220^{\circ} \mathrm{C}$. Ion scanning range $100-1000 \mathrm{~m} / \mathrm{z}$; rolling average spectral ratio $3 * 1.00 \mathrm{~Hz}$. Dp-3-O-glu, Pt-3-O-glu, and Mv-3-O-glu are used as standards to calculate the content of these three anthocyanins. Others use Mv-3-O-glu as the equivalent to calculate its content.

\subsection{Color of Wines}

The color of the wine was determined according to the CIE $\mathrm{L} * \mathrm{a} * \mathrm{~b} *$ color space method. Using a precision colorimeter (Shenzhen WAVE Optoelectronics Technology Co., Ltd.) to automatically compare the color difference between the sample and the tested sample and output three sets of CIE L $* \mathrm{a} * \mathrm{~b} *$ data. The three components $\mathrm{L} * \mathrm{a} *$, and $\mathrm{b} *$ are digitized the color change of wines [31-35]. Calculate the color difference of wine according to the uniform color space formula.

$$
\Delta \mathrm{E}=\sqrt{(\Delta \mathrm{L})^{2}+(\Delta \mathrm{a})^{2}+(\Delta \mathrm{b})^{2}}
$$

The wine sample was filtered through a $0.45 \mu \mathrm{m}$ aqueous filter. Remove the protective lens cap and turn on the switch. Align the lens with the standard white cavity and standard black cavity for calibration. Take $20 \mathrm{ml}$ sample into the multi-function measurement cavity, place the lens directly on the sample for measurement, and then press the work key. After hearing a "drop" sound, remove the lens and record the reading. 


\subsection{Statistical Analysis}

Data were expressed as mean \pm standard deviation. Student's-test was performed for the comparison of the means between control and treated groups. The statistical procedure was carried out with SPSS Version 22.0statistical package for Windows (Chicago, IL). The data for the different determinations were processed using the variance analysis (ANOVA). Bruker compass Date Analysis 4.4 SR1 was used to analyze the liquid quality data.

\section{Results and Discussion}

\subsection{Berry and Wine Attributes}

To examined the effect of ABA and NAA on the development of grape berries, the soluble solids, total acidity, $\mathrm{pH}$, and berries weight of the grapes were determined. As shown in Table 1, ABA treatment significantly increased berry weight, total soluble solids and $\mathrm{pH}$ of the berries. And NAA treatment decreased the physicochemical indices of berries (Table 1).

TABLE 1. General parameters of grape and wine for different treatments

\begin{tabular}{ccccc}
\hline Treatments & $\begin{array}{c}\text { Total soluble solids } \\
\left({ }^{\circ} \text { Brix }\right)\end{array}$ & $\begin{array}{c}\text { Titratable acidity } \\
(\mathrm{g} / \mathrm{L})\end{array}$ & $\mathrm{pH}$ & $\begin{array}{c}\text { Berry Weight } \\
(\mathrm{g})\end{array}$ \\
\hline Grapes & & & & \\
control & $22.5 \pm 0.1 \mathrm{~b}$ & $6.68 \pm 0.04 \mathrm{~b}$ & $3.52 \pm 0.03 \mathrm{~b}$ & $124.17 \pm 0.93 \mathrm{~b}$ \\
$1000 \mathrm{mg} / \mathrm{L}$ & $23 \pm 0.3 \mathrm{a}$ & $6.50 \pm 0.02 \mathrm{c}$ & $3.64 \pm 0.04 \mathrm{a}$ & $126.02 \pm 1.05 \mathrm{a}$ \\
ABA & $22 \pm 0.1 \mathrm{c}$ & $6.76 \pm 0.05 \mathrm{a}$ & $3.46 \pm 0.04 \mathrm{~b}$ & $121.63 \pm 0.98 \mathrm{c}$ \\
\hline $200 \mathrm{mg} / \mathrm{L} \mathrm{NAA}$ & Total sugar $(\mathrm{g} / \mathrm{L})$ & Titratable acid $(\mathrm{g} / \mathrm{L})$ & $\mathrm{pH}$ & Alcohol $(\%$ vol. $)$ \\
\hline Treatments & & & & \\
\hline Wines & $1.06 \pm 0.03 \mathrm{ab}$ & $4.2 \pm 0.03 \mathrm{ab}$ & $3.69 \pm 0.03 \mathrm{a}$ & $14.3 \pm 0.03 \mathrm{~b}$ \\
control & $1.07 \pm 0.06 \mathrm{a}$ & $4.17 \pm 0.03 \mathrm{~b}$ & $3.66 \pm 0.03 \mathrm{ab}$ & $14.5 \pm 0.03 \mathrm{a}$ \\
$1000 \mathrm{mg} / \mathrm{L}$ & $1.04 \pm 0.04 \mathrm{~b}$ & $4.25 \pm 0.03 \mathrm{a}$ & $3.64 \pm 0.03 \mathrm{~b}$ & $14.3 \pm 0.03 \mathrm{~b}$ \\
ABA & &
\end{tabular}

Different letters in the same column indicate significant differences between means ( $\mathrm{p}<0.05)$.

This may be because NAA delayed fruit maturation and sugar accumulation. As for the titratable acid content in berries, the ABA treatment group decreased by $2.69 \%$, which was significantly lower than that of the control group, while the NAA treatment group was significantly higher than control by $1.2 \%$. For wine, the alcohol content was significantly increased by ABA treatment and NAA decreased the $\mathrm{pH}$ of the wine. But there was no significant effect on the total sugar and titratable acid content in the wine after both treatments (Table 1).

\subsection{Total Phenolics in Grape Skins}

As Fig. 1 showed, the total phenolic content gradually decreased from the E-L 35 stage to the E-L37 stage. Then it started to accumulate and reached the maximum at the E-L 38 stage. 


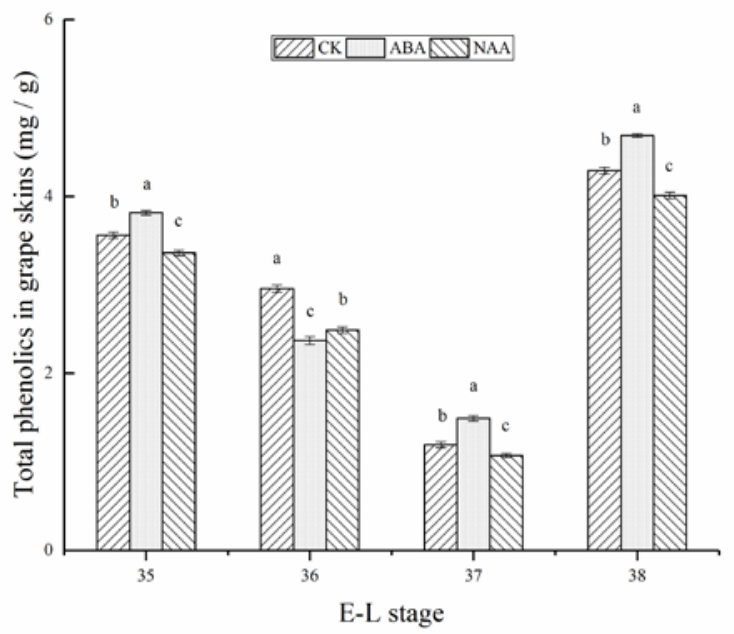

Figure 1. Total phenolics content in peel at different stages.

At harvest, the total phenolic content of ABA treatment was significantly higher than the control group. And NAA treatment did the opposite. ABA treatment significantly increased the accumulation of total phenolic in the grape skins, while NAA had the opposite effect. It shows that ABA has played a positive role in accelerating the accumulation of phenolics during the maturation and development of the process [36-41], while NAA significantly inhibits the synthesis of phenolics in berries [42] (Fig. 1).

\subsection{Total Flavonoids in Grape Skins}

The contents of flavonoids in the grape skins showed an increase from E-L 35 stage to the E-L37 stage and then a decrease from E-L37 to the E-L 38 stage (Fig. 2).

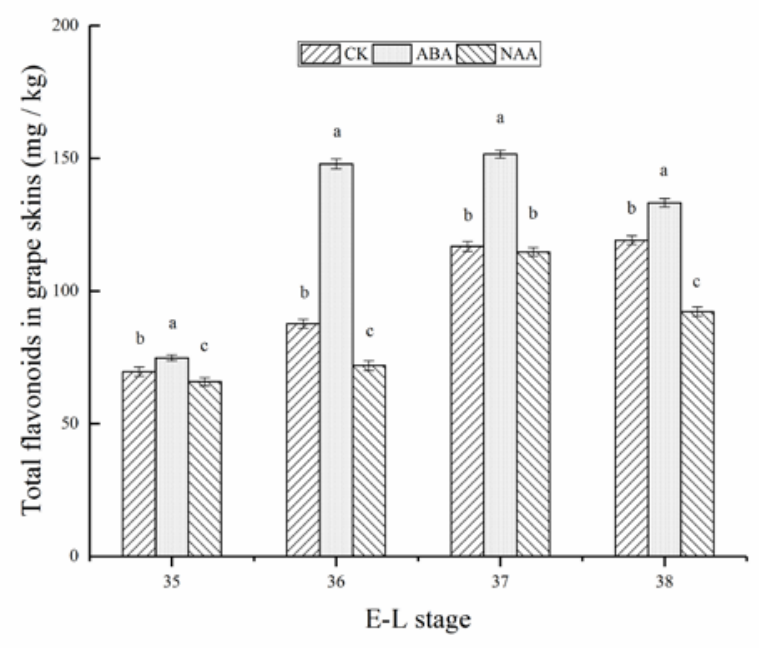

Figure 2. Total flavonoids content in peel at different stages.

But the overall trend was still increasing. The flavonoids of the control group accumulated slowly. The ABA treatment accumulated rapidly at E-L 36 and reached a peak at the E-L 37 stage. At this time, the contents reached $151.58 \mathrm{mg} / \mathrm{kg}$, after which the contents began to decrease slowly, and the final contents reached $133.25 \mathrm{mg} / \mathrm{kg}$. The contents of flavonoids in the NAA treatment group was always the lowest in the three groups. The highest content was $114.72 \mathrm{mg} / \mathrm{kg}$, and at harvest the 
contents decreased to $92.19 \mathrm{mg} / \mathrm{kg}$. The final increase rates of the three treatment groups were $71.17 \%, 78.14 \%$, and $40.20 \%$. Compared with the control group, ABA treatment significantly increased the flavonoid content, while NAA treatment significantly reduced the flavonoid content.

The application of elicitors and stimulating agents belong to a method of viticulture practice to improve the quality of grape berries. In this study, the total phenolic contents and flavonoids in the skins of the V. vinifera wine grapes were significantly enhanced by ABA treatment. The result was similar to a previous research for a muscadine grape 'Noble' [39]. Grape berry ripening and harvest can be delayed by the application of NAA during the pre-veraison period of berry development. In this case, a considerable delay in harvest was achieved using NAA.

\subsection{Total Anthocyanin Content in Grape Skins}

During the development and maturation of grapes, the content of anthocyanins in berries under different treatments showed an increasing trend. With the accumulation of anthocyanins, the berries gradually softened and colored. The accumulation of anthocyanins in the berry skins was enhanced by ABA treatment and suppressed by NAA from the E-L 35 to E-L 37 stage. But at harvest, the content of anthocyanins in the control group reached $4.85 \mathrm{mg} / \mathrm{g}$, while the anthocyanin under ABA treatment is $4.71 \mathrm{mg} / \mathrm{g}$. It did not increase the accumulation of total anthocyanins compared with the control (Figure 3).

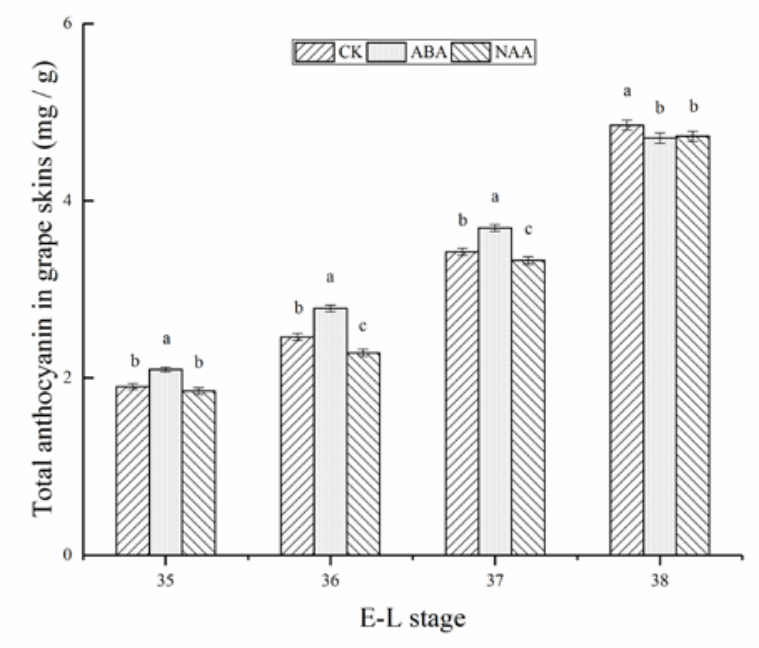

Figure 3. Total anthocyanins content in peel at different stages.

ABA can promote the expression of anthocyanin-synthesis-related genes, and can promote berries coloring during the ripening and development [37, 41]. But did not increase the content of total anthocyanins in berries [43]. The result indicated the ABA-treated berries were overripe at harvest. Grapes from ABA treatment significantly underwent a faster color accumulation in grape skin compared to control. The accumulation of anthocyanin in grapes treated with NAA was slowest. It reached $4.73 \mathrm{mg} / \mathrm{g}$ at harvest, which was significantly lower than that of the control (Fig. 3). NAA treatment not only delays the ripening and coloring rate of the berries, but also inhibits the accumulation of anthocyanins [43-45].

\subsection{Total Anthocyanin Contents and Anthocyanins Profiles in Wines}

Anthocyanins affect the color and stability of wine directly. The content of anthocyanin in wine has an important influence on the sensory evaluation and quality of wine. Total anthocyanin contents were significantly lower in both ABA and NAA treatments groups than that of control group (Fig. 4), which is in accordance with the content of anthocyanin in grape skins. 


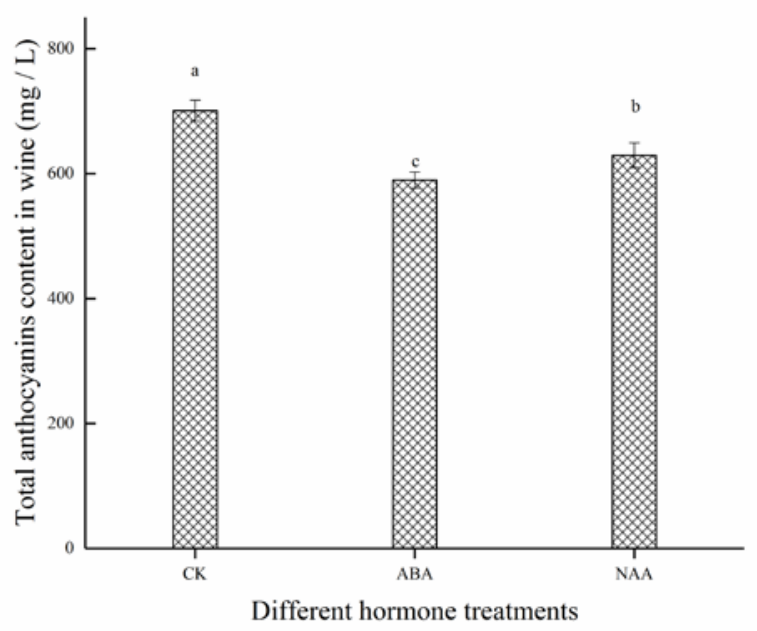

Figure 4. Total anthocyanins content in wine with different treatments.

Based on the UV-visible spectrum of anthocyanins, MS/MS characteristic ions, fragment ions, retention time and literature analysis [46-50], we detected 19 individual anthocyanins in wine samples (Figure 5). According to substituent, acylated form, and elution order of the pyran polymer, the nineteen individual anthocyanins include seven un-acylated individual anthocyanins, seven acetylated individual anthocyanins, four coumarin acylated individual anthocyanins and one coffee acylated individual anthocyanins (Table 2).

TABLE 2. Chromatographic data (peak number and retention time) and MS2 m/z values (molecular and fragment ions) content of anthocyanins detected in wine

\begin{tabular}{|c|c|c|c|c|c|c|c|}
\hline \multirow{2}{*}{$\begin{array}{c}\text { Numbe } \\
r\end{array}$} & \multirow[t]{2}{*}{ Compound } & \multirow{2}{*}{$\begin{array}{c}\mathrm{MS}(\mathrm{m} / \mathrm{z} \\
)\end{array}$} & \multirow{2}{*}{$\begin{array}{c}\mathrm{MS} / \mathrm{MS}(\mathrm{m} / \mathrm{z} \\
)\end{array}$} & \multirow{2}{*}{$\begin{array}{l}\mathrm{RT} \\
(\mathrm{min} \\
\text { ) }\end{array}$} & \multicolumn{3}{|c|}{ Anthocyanin content (mg / L) } \\
\hline & & & & & CK & ABA & NAA \\
\hline 1 & Dp-3-O-glu & 465 & 303 & 5.7 & 19.4385 & 13.6883 & 30.4083 \\
\hline 2 & Cy-3-O-glu & 449 & 287 & 6.1 & 0.2232 & 0.1797 & 0.6611 \\
\hline 3 & Pt-3-O-glu & 479 & 317 & 6.7 & 31.9359 & 24.3793 & 48.92 \\
\hline 4 & Pn-3-O-glu & 463 & 301 & 7.2 & 4.2106 & 4.4304 & 7.2565 \\
\hline 5 & Mv-3-O-glu & 493 & 331 & 8.1 & $\begin{array}{c}312.061 \\
6\end{array}$ & $\begin{array}{c}243.434 \\
5\end{array}$ & $\begin{array}{c}278.725 \\
7\end{array}$ \\
\hline 6 & Dp-3-O-acetylglu & 507 & 303 & 9 & 3.933 & 2.127 & 6.1923 \\
\hline 7 & Cy-3-O-acetylglu & 491 & 287 & 9.5 & 0.5152 & 0.5874 & 2.3254 \\
\hline 8 & $\begin{array}{l}\text { Pt-3-O-acetylglu } \\
\text { 4-vinylformic acid }\end{array}$ & 521 & 317 & 9.8 & 9.5636 & 6.3577 & 15.7451 \\
\hline 9 & $\begin{array}{c}\text { adduct of } \\
\text { Mv-3-O-acetylglu }\end{array}$ & 603 & 339 & 10.1 & 1.9028 & 0.9112 & 0.7101 \\
\hline 10 & Pn-3-O-acetylglu & 505 & 301 & 10.4 & 10.3787 & 14.777 & 24.1219 \\
\hline 11 & Mv-3-O-acetylglu & 535 & 331 & 10.9 & 254.36 & $\begin{array}{c}165.379 \\
3\end{array}$ & $\begin{array}{c}209.635 \\
7\end{array}$ \\
\hline 12 & Mv-3-O-caffeoylglu & 655 & 331 & 11.6 & 1.7813 & 1.3074 & 1.677 \\
\hline 13 & Pt-3-O-coumaroylglu & 625 & 317 & 11.9 & 1.1945 & 1.2314 & 2.8781 \\
\hline 14 & $\begin{array}{c}\text { Pn-3-O-coumaroylgl } \\
\text { u }\end{array}$ & 609 & 301 & 12.5 & 4.0524 & 5.2243 & 7.3049 \\
\hline 15 & Mv-3-O-coumaroylgl & 639 & 331 & 12.8 & 40.729 & 38.1269 & 36.0559 \\
\hline
\end{tabular}




$$
\text { 4-vinylphenol adduct }
$$

609

4-vinylphenol adduct of Mv-3- O-acetylglu

651 4-vinylphenol adduct

The main type of anthocyanins found was anthocyanidin 3-glucosides (Table 2 and Fig. 5), the most important one being derived from malvidin followed by other common grape anthocyanidins (Delphinidin, Cyanidin, Petunidinand Peonidin).

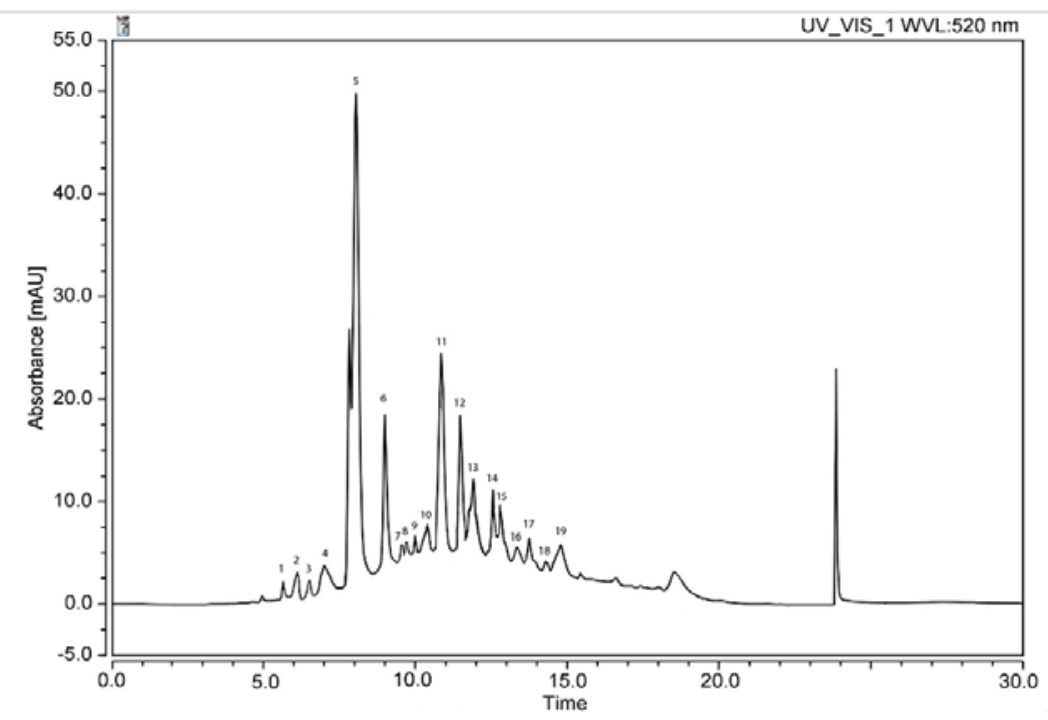

Figure 5. Chromatogram of wine recorded at $520 \mathrm{~nm}$.

In terms of the proportion of species, anthocyanins in wine are mainly composed of 5 basic anthocyanin monomers glucosides, acetylated glucosides, and coumalylated glucosides. And the content of coffee acylated glucoside is very small or even negligible.

Among the three treatments, the control accounted for $46.95 \%$ of the acylated individual anthocyanins. The ABA treatment group was $44.97 \%$ and the NAA treatment group was $45.44 \%$. Compared with the control group, both ABA and NAA treatment reduced the proportion of acylated anthocyanins in wine. As for the ABA and NAA treatment groups, there was no significant difference in the proportion of total acylated anthocyanins. But the content of acetylated individual anthocyanins in the ABA treatment was lower than that in the NAA treatment group. And the content of coumalylated individual anthocyanins in the ABA treatment group was higher than NAA treatment group (Table 3).

TABLE 3. Proportion of anthocyanins in wine with different treatments

\begin{tabular}{ccccc}
\hline Treatments & Un-acylated & \multicolumn{3}{c}{ Acylated } \\
\cline { 3 - 5 } & & Acetylation & Acetylation & Acetylation \\
\hline CK & $53.05 \%$ & $40.11 \%$ & $0.25 \%$ & $6.58 \%$ \\
ABA & $55.03 \%$ & $36.24 \%$ & $0.25 \%$ & $8.48 \%$ \\
NAA & $54.56 \%$ & $38.37 \%$ & $0.25 \%$ & $6.83 \%$ \\
\hline
\end{tabular}

For the content ratio, malvidin glucosides, as the main individual anthocyanins in wine, accounted for $87.91 \%, 86.22 \%, 78.53 \%$ of the total anthocyanins in the three treatments. Mv-acoccupied the 
most content of acylated anthocyanin in wine which is acylated by $\mathrm{Mv}$ and anthocyanin acyl transferase [51]. It was significantly reduced by NAA treatments. The content of petunidin glucosides was $6.04 \%$, 6.04\%, 9.94\%. Delphinidin glucosides were $3.31 \%$, 2.99\% and 5.39\%. Peonidin glucosides were $2.64 \%, 4.61 \%$ and $5.69 \%$ in the three treatments. The content of cyanidin glucosides are extremely small or even negligible (Table 2).

\subsection{Wine Color}

The results show that after one year of aging, $\Delta \mathrm{L}$ is a positive value, which indicates that the color of the wine sample has changed to a white tone. And $\Delta \mathrm{L}$ (NAA) $>\Delta \mathrm{L}$ (ABA) $>\Delta \mathrm{L}$ (CK), indicated that NAA and ABA have greater color changes than the control group. $\Delta \mathrm{a}$ is negative, indicating that the wine sample is changing to a green tone. $\Delta \mathrm{a}(\mathrm{ABA}) \Delta \mathrm{a}(\mathrm{NAA})>\Delta \mathrm{a}(\mathrm{CK})$, indicated that ABA and NAA treatments have more obvious changes to the green tone than the control group.

TABLE 4. CIE $\mathrm{L} * \mathrm{a} * \mathrm{~b} *$ measures the color change of wine

\begin{tabular}{|c|c|c|c|c|c|c|c|c|c|c|}
\hline \multirow[t]{2}{*}{ Sample } & \multicolumn{3}{|c|}{ Fresh wine } & \multicolumn{3}{|c|}{ One year later } & \multirow[b]{2}{*}{$\triangle \mathrm{L}$} & \multirow[b]{2}{*}{$\triangle \mathrm{a}$} & \multirow[b]{2}{*}{$\triangle \mathrm{b}$} & \multirow[b]{2}{*}{$\triangle \mathrm{E}$} \\
\hline & $\mathrm{L}$ & $\mathrm{a}$ & $\mathrm{b}$ & $\mathrm{L}$ & $\mathrm{a}$ & $\mathrm{b}$ & & & & \\
\hline CK & 22.37 & 4.98 & -6.04 & 23.59 & 4.21 & -5.36 & 1.22 & -0.77 & 0.68 & 1.59 \\
\hline ABA & 22.21 & 4.75 & -5.36 & 23.57 & 3.64 & -4.55 & 1.36 & -1.11 & 0.81 & 1.93 \\
\hline NAA & 22.09 & 4.63 & -5.96 & 23.69 & 3.6 & -4.94 & 1.6 & -1.03 & 1.02 & 2.16 \\
\hline
\end{tabular}

Note: L means black and white; a means red and green; b means yellow and blue

$\Delta \mathrm{b}$ is a positive value indicating that the wine sample is changing to a yellow tone. $\Delta \mathrm{b}$ (NAA) $>\Delta \mathrm{b}$ (ABA) $>\Delta \mathrm{b}(\mathrm{CK})$ indicated that NAA and ABA treatments have a more pronounced transition to yellow tones. $\Delta \mathrm{E}$ is the color difference value. $\Delta \mathrm{E}$ (NAA) $>\Delta \mathrm{E}$ (ABA) $>\Delta \mathrm{E}$ (CK), indicated that the rate of color oxidation decay of wine samples treated with NAA and ABA was faster than that of the control group. Based on Table 4, there is no significant difference in the ratio of acylated anthocyanins in NAA and ABA treatment groups. The reason that why wine color oxidized faster with NAA treated than ABA treated may be due to the higher content of coumalylated anthocyanins in ABA treatment. Studies have shown that coumalylation is more stable than acetylation [52-56].

\section{Conclusion}

In this study, the effect of exogenous ABA and NAA on phenolic characteristics of V. vinifera grape cultivar 'Cabernet Sauvignon'were systematically studied. The research materials covered the skins of grape berries as well as the wines. The phenolic characteristics included the content of phenolic compounds (total phenolics, anthocyanins and flavonols) of grape skins and composition of wine anthocyanins, which were important to the organoleptic properties and nutritional values of grape berries and wines. In conclusion, ABA treatment significantly increased berry weight, total soluble solids and $\mathrm{pH}$ of the berries. And NAA treatment decreased the physicochemical indices of berries. Exogenous ABA applied prior veraison could enhance phenolic contents of grape skins. But at harvest, the accumulation of anthocyanins under ABA treatment was lower than control. This maybe because the $1000 \mathrm{mg} / \mathrm{L} \mathrm{ABA}$ spray promoted grape maturity. the ABA-treated berries were overripe at harvest. Grapes from ABA treatment significantly underwent a faster color accumulation in grape skin. And NAA treatment did the opposite. The result had practical significance for the application of exogenous ABA in viticulture of wine grapes. We detected 19 individual anthocyanins in wine samples of the three treatment. Both ABA and NAA treatment reduced the proportion of acylated anthocyanins in wine, which led to the rate of color oxidation decay of wine samples treated with NAA and ABA was faster than that of the control group. So maybe the concentration of ABA spray is too high in this experiment. Because the phenolic contents and compositions of grapes and wines are influenced by many environmental factors in field, such as light, temperature, rainfall and biotic stress. Furthermore, more researches are needed into the molecular mechanisms of exogenous 
ABA and NAA on the phenolic content of grape skins, and also into the effect of the changes in anthocyanin composition on color stability of wines.

\section{Acknowledgements}

This research was supported by grants from the Shandong Modern Agricultural Industry Technology System Fruit Industry Innovation Team (SDAIT-06-14).

\section{References}

[1] He, F., et al., Chemical synthesis of proanthocyanidins in vitro and their reactions in aging wines. 2008. 13(12): p. 3007-3032.

[2] Castellarin, S.D., et al., Colour variation in red grapevines (Vitis vinifera L.): genomic organisation, expression of flavonoid 3'-hydroxylase, flavonoid 3', 5'-hydroxylase genes and related metabolite profiling of red cyanidin-/blue delphinidin-based anthocyanins in berry skin. 2006. 7(1): p. 12.

[3] Jeong, S., et al., Expression of the flavonoid $3^{\prime}$-hydroxylase and flavonoid 3' , 5' -hydroxylase genes and flavonoid composition in grape (Vitis vinifera). 2006. 170(1): p. 61-69.

[4] Koes, R., W. Verweij, and F.J.T.i.p.s. Quattrocchio, Flavonoids: a colorful model for the regulation and evolution of biochemical pathways. 2005. 10(5): p. 236-242.

[5] Pomar, F., M. Novo, and A.J.J.o.C.a. Masa, Varietal differences among the anthocyanin profiles of 50 red table grape cultivars studied by high performance liquid chromatography. 2005. 1094(1-2): p. 34-41.

[6] Flamini, R., et al., Advanced knowledge of three important classes of grape phenolics: anthocyanins, stilbenes and flavonols. 2013. 14(10): p. 19651-19669.

[7] Castañeda-Ovando, A., et al., Chemical studies of anthocyanins: A review. 2009. 113(4): p. 859-871.

[8] de Pascual-Teresa, S. and M.T.J.P.r. Sanchez-Ballesta, Anthocyanins: from plant to health. 2008. 7(2): p. 281-299.

[9] Núñez, V., et al., Vitis vinifera L. cv. Graciano grapes characterized by its anthocyanin profile. 2004. 31(1): p. 69-79.

[10] Azevedo, J., et al., Effect of sugar acylation on the antioxidant properties of Vitis vinifera red grape malvidin - 3 - glucoside. 2011. 46(2): p. 343-349.

[11] Brouillard, R., et al., The co-pigmentation reaction of anthocyanins: a microprobe for the structural study of aqueous solutions. 1989. 111(7): p. 2604-2610.

[12] Lecourieux, F., et al., An update on sugar transport and signalling in grapevine. 2014. 65(3): p. 821-832.

[13] HUANG, X.M., H.B.J.A.J.o.G. HUANG, and W. Research, Early post - veraison growth in grapes: evidence for a two - step mode of berry enlargement. 2001. 7(3): p. 132-136.

[14] Garrido, J. and F.J.F.r.i. Borges, Wine and grape polyphenols-A chemical perspective. 2013. 54(2): p. 1844-1858.

[15] Agudelo-Romero, P., et al., Study of polyamines during grape ripening indicate an important role of polyamine catabolism. 2013. 67: p. 105-119.

[16] Kühn Weber, N.A., Berry ripening: recently heard through the grapevine. 2014. 
[17] Koyama, R., et al., Abscisic Acid Application Affects Color and Acceptance of the New Hybrid 'BRS Melodia'Seedless Grape Grown in a Subtropical Region. 2019. 54(6): p. 1055-1060.

[18] Koyama, R., et al., Exogenous abscisic acid increases the anthocyanin concentration of berry and juice from 'Isabel'grapes (Vitis labrusca L.). 2014. 49(4): p. 460-464.

[19] Neto, F.J.D., et al., Effect of ABA on colour of berries, anthocyanin accumulation and total phenolic compounds of'Rubi'table grape ('Vitis vinifera'). 2017. 11(2): p. 199.

[20] Yamamoto, L.Y., et al., Color of berry and juice of'Isabel'grape treated with abscisic acid in different ripening stages. 2015. 50(12): p. 1160-1167.

[21] Zhu, L., et al., Effects of exogenous abscisic acid on phenolic characteristics of red Vitis vinifera grapes and wines. 2016. 25(2): p. 361-370.

[22] Cecilia Peppi, M., M.W. Fidelibus, and N.K.J.I.J.o.F.S. Dokoozlian, Timing and concentration of abscisic acid applications affect the quality of 'Crimson Seedless' grapes. 2008. 7(4): p. 71-83.

[23] Peppi, M., et al., Application timing and concentration of abscisic acid affect the quality of 'Redglobe'grapes. 2007. 82(2): p. 304-310.

[24] Peppi, M.C., M.W. Fidelibus, and N.J.H. Dokoozlian, Abscisic Acid Application Timing and Concentration Affect Firmness, Pigmentation, and Color ofFlame Seedless' Grapes. 2006. 41(6): p. 1440-1445.

[25] Ziliotto, F., et al., Grape berry ripening delay induced by a pre-véraison NAA treatment is paralleled by a shift in the expression pattern of auxin-and ethylene-related genes. 2012. 12(1): p. 185.

[26] 26. Gouthu, S., et al., A comparative study of ripening among berries of the grape cluster reveals an altered transcriptional programme and enhanced ripening rate in delayed berries. 2014. 65(20): p. 5889-5902.

[27] Coombe, B.J.A.j.o.g. and w. research, Growth stages of the grapevine: adoption of a system for identifying grapevine growth stages. 1995. 1(2): p. 104-110.

[28] Cliff, M.A., M.C. King, and J.J.F.R.I. Schlosser, Anthocyanin, phenolic composition, colour measurement and sensory analysis of BC commercial red wines. 2007. 40(1): p. 92-100.

[29] Minussi, R.C., et al., Phenolic compounds and total antioxidant potential of commercial wines. 2003. 82(3): p. 409-416.

[30] Mattivi, F., et al., Metabolite profiling of grape: flavonols and anthocyanins. 2006. 54(20): p. 7692-7702.

[31] Gonnet, J.-F.J.J.o.h.s., CIELab measurement, a precise communication in flower colour: an example with carnation (Dianthus caryophyllus) cultivars. 1993. 68(4): p. 499-510.

[32] Gonnet, J.-F.J.F.C., Colour effects of co-pigmentation of anthocyanins revisited-1. A colorimetric definition using the CIELAB scale. 1998. 63(3): p. 409-415.

[33] Gonnet, J.-F.J.F.C., Colour effects of co-pigmentation of anthocyanins revisited-2. A colorimetric look at the solutions of cyanin co-pigmented byrutin using the CIELAB scale. 1999. 66(3): p. 387-394.

[34] Gonnet, J.-F.J.F.c., Colour effects of co-pigmentation of anthocyanin revisited-3. A further description using CIELAB differences and assessment of matched colours using the CMC model. 2001. 75(4): p. 473-485.

[35] Hill, B., T. Roger, and F.W.J.A.T.o.G. Vorhagen, Comparative analysis of the quantization of color spaces on the basis of the CIELAB color-difference formula. 1997. 16(2): p. 109-154. 
[36] Berli, F.J., et al., Solar UV-B and ABA are involved in phenol metabolism of Vitis vinifera L. increasing biosynthesis of berry skin polyphenols. 2011. 59(9): p. 4874-4884.

[37] Jiang, Y. and D.C.J.P.G.R. Joyce, ABA effects on ethylene production, PAL activity, anthocyanin and phenolic contents of strawberry fruit. 2003. 39(2): p. 171-174.

[38] Khaleghnezhad, V., et al., Interactive effects of abscisic acid and temperature on rosmarinic acid, total phenolic compounds, anthocyanin, carotenoid and flavonoid content of dragonhead (Dracocephalum moldavica L.). 2019. 250: p. 302-309.

[39] Sandhu, A.K., et al., Effects of exogenous abscisic acid on antioxidant capacities, anthocyanins, and flavonol contents of muscadine grape (Vitis rotundifolia) skins. 2011. 126(3): p. 982-988.

[40] Thiruvengadam, M., S.-H. Kim, and I.-M.J.S.H. Chung, Exogenous phytohormones increase the accumulation of health-promoting metabolites, and influence the expression patterns of biosynthesis related genes and biological activity in Chinese cabbage (Brassica rapa spp. pekinensis). 2015. 193: p. 136-146.

[41] Wu, Q., et al., Contribution of abscisic acid to aromatic volatiles in cherry tomato (Solanum lycopersicum L.) fruit during postharvest ripening. 2018. 130: p. 205-214.

[42] Prasad, M., J.G.J.J.o.P.B. Satyasree, and Biotechnology, $\alpha$-Naphthalene Acetic Acid Regulated Anthocyanins, Polyphenols and Associated Enzymes in Euphorbia heterophylla L. Seedling Explant Cultures. 1994. 3(2): p. 69-72.

[43] Jeong, S.T., et al., Effects of plant hormones and shading on the accumulation of anthocyanins and the expression of anthocyanin biosynthetic genes in grape berry skins. 2004. 167(2): p. 247-252.

[44] Ali, M., B.H.J.A.b. Abbasi, and biotechnology, Thidiazuron-induced changes in biomass parameters, total phenolic content, and antioxidant activity in callus cultures of Artemisia absinthium L. 2014. 172(5): p. 2363-2376.

[45] Liu, Z., M.-Z. Shi, and D.-Y.J.P. Xie, Regulation of anthocyanin biosynthesis in Arabidopsis thaliana red pap1-D cells metabolically programmed by auxins. 2014. 239(4): p. 765-781.

[46] Downey, M.O. and S.J.J.o.C.A. Rochfort, Simultaneous separation by reversed-phase high-performance liquid chromatography and mass spectral identification of anthocyanins and flavonols in Shiraz grape skin. 2008. 1201(1): p. 43-47.

[47] Li, S.Y., et al., A systematic analysis strategy for accurate detection of anthocyanin pigments in red wines. 2016. 30(13): p. 1619-1626.

[48] Liang, Z., et al., Anthocyanin composition and content in grape berry skin in Vitis germplasm. 2008. 111(4): p. 837-844.

[49] Quaglieri, C., et al., Comparison of Aquitaine and rioja red wines: Characterization of their phenolic composition and evolution from 2000 to 2013. 2017. 22(2): p. 192.

[50] Wang, H., et al., Characterization of anthocyanins in grape juices by ion trap liquid chromatography- mass spectrometry. 2003. 51(7): p. 1839-1844.

[51] Nakayama, T., H. Suzuki, and T.J.J.o.M.C.B.E. Nishino, Anthocyanin acyltransferases: specificities, mechanism, phylogenetics, and applications. 2003. 23(2-6): p. 117-132.

[52] Dangles, O., N. Saito, and R.J.P. Brouillard, Anthocyanin intramolecular copigment effect. 1993. 34(1): p. 119-124.

[53] Figueiredo, P., et al., New aspects of anthocyanin complexation. Intramolecular copigmentation as a means for colour loss? 1996. 
[54] Figueiredo, P., et al., New features of intramolecular copigmentation byacylated anthocyanins. 1999. 51(1): p. 125-132.

[55] George, F., et al., Influence of trans-cis isomerisation of coumaric acid substituents on colour variance and stabilisation in anthocyanins. 2001. 57(5): p. 791-795.

[56] Han, F.-L., et al., Principal component regression analysis of the relation between CIELAB color and monomeric anthocyanins in young Cabernet Sauvignon wines. 2008. 13(11): p. 2859-2870. 